\title{
The NO/cGMP pathway inhibits transient cAMP signals through the activation of PDE2 in striatal neurons
}

\section{Marina Polito ${ }^{1,2}$, Jeffrey Klarenbeek ${ }^{3}$, Kees Jalink ${ }^{3}$, Danièle Paupardin-Tritsch ${ }^{1,2}$, Pierre Vincent ${ }^{1,2 * t}$ and Liliana R. V. Castro ${ }^{1,2 t}$}

1 UMR7102, Centre National de la Recherche Scientifique, Paris, France

2 UMR7102, Neurobiology of Adaptive Processes, Université Pierre et Marie Curie, Paris, France

${ }^{3}$ Cellbiophysics Group, The Netherlands Cancer Institute, Amsterdam, Netherlands

\section{Edited by:}

Arianna Maffei, SUNY Stony Brook, USA

\section{Reviewed by:}

John Garthwaite, University College London, UK

Gang Wang, Weill Cornell Medical

College, USA

\section{*Correspondence:}

Pierre Vincent, Centre National de la Recherche Scientifique, UMR7102,

9 quai St Bernard F-75005, Paris,

France

e-mail:pierre.vincent@upmc.fr

these authors have contributed equally to this work.
The NO-cGMP signaling plays an important role in the regulation of striatal function although the mechanisms of action of cGMP specifically in medium spiny neurons (MSNs) remain unclear. Using genetically encoded fluorescent biosensors, including a novel Epac-based sensor (EPAC-S ${ }^{H 150}$ ) with increased sensitivity for CAMP, we analyze the cGMP response to NO and whether it affected cAMP/PKA signaling in MSNs. The Cygnet2 sensor for cGMP reported large responses to NO donors in both striatonigral and striatopallidal MSNs, this CGMP signal was controlled partially by PDE2. At the level of CAMP brief forskolin stimulations produced transient CAMP signals which differed between $D_{1}$ and $D_{2}$ MSNs. NO inhibited these cAMP transients through cGMP-dependent PDE2 activation, an effect that was translated and magnified downstream of cAMP, at the level of PKA. PDE2 thus appears as a critical effector of NO which modulates the post-synaptic response of MSNs to dopaminergic transmission.

Keywords: cyclic AMP, cyclic GMP, phosphodiesterase, biosensor imaging, nitric oxide, striatum, dopamine

\section{INTRODUCTION}

Cyclic nucleotides control a range of cellular processes, particularly in neurons where they transduce extracellular signals carried by neuromodulators. The striatum is involved in reward, motor control and action selection, and cyclic nucleotide signaling plays a critical role in the normal function of this brain structure. While the involvement of cAMP in striatal physiology is widely acknowledged, much less is known on cGMP, although this signaling cascade also plays a critical role in the regulation of striatal function (West and Tseng, 2011). Medium spiny neurons (MSNs) in the striatum constitute $95 \%$ of the neuronal types, approximately half of which are anatomically defined as the "direct pathway" and express dopamine type 1 receptors $\left(D_{1}\right)$. This receptor is positively coupled to cAMP production. The other half of MSNs, defined as the "indirect pathway," express high levels of dopamine type 2 receptors $\left(D_{2}\right)$ and adenosine $A_{2 A}$ receptors (Le Moine and Bloch, 1995; Bateup et al., 2008; Bertran-Gonzalez et al., 2008). $D_{2}$ receptors are negatively coupled to adenylyl cyclases (AC) and therefore inhibit cAMP production while the $\mathrm{A}_{2 \mathrm{~A}}$ receptors are positively coupled to $\mathrm{AC}$ and increase cAMP levels. The segregation between $D_{1}$ and $A_{2 A}$ expressing neurons is clearly visualized using cAMP/PKA biosensors (Castro et al., 2013). The functional effects of the cAMP signaling cascade has been widely documented in the striatum (Hervé and Girault, 2005) but much less is know about the cGMP signaling. cGMP is produced by the $\mathrm{NO}$ receptor, aka soluble guanylyl cyclase (sGC), in response to nitric oxide (NO), and NO/cGMP signaling regulates a number of neurobiological processes (Garthwaite, 2008). sGC is highly expressed in the striatum (Ariano et al., 1982; Matsuoka et al., 1992; Ding et al., 2004) while the NO producing enzyme
nNOS is highly expressed by a fraction of striatal interneurons (Vincent and Kimura, 1992; Rushlow et al., 1995; Kawaguchi, 1997; Vincent, 2000). It is commonly accepted that NO produced by NOS interneurons diffuses throughout the striatal complex and increases corticostriatal and dopaminergic synaptic transmission via a sGC-cGMP dependent mechanism (West et al., 2002; West and Grace, 2004; West and Tseng, 2011). In addition, NO diffuses into the dendrites of MSNs modulating corticostriatal synaptic plasticity in vitro (Calabresi et al., 1999, 2000) and in vivo (West and Grace, 2004). While these effects are clearly mediated by cGMP, the downstream effectors of cGMP, often assumed to be the cGMP-dependent protein kinase (PKG), remain uncertain, since only moderate levels of expression have been reported in the striatum (el-Husseini et al., 1995; El-Husseini et al., 1999; de Vente et al., 2001) and CNG expression has not been reported in the striatum (Wei et al., 1998).

An important part of the signal transduction process is the rapid degradation of the cyclic nucleotides by cyclic nucleotide phosphodiesterases (PDEs). PDE2 mRNA and protein are present at high levels in the striatum (Repaske et al., 1993; Van Staveren et al., 2003) and functionally active (Wykes et al., 2002; Lin et al., 2010). PDE2 has dual enzymatic activity allowing to hydrolyze both cAMP and cGMP (Erneux et al., 1981; Martins et al., 1982). A characteristic feature of PDE2 is the positive cooperativity of the substrate cGMP: in the absence of cGMP, PDE2 activity is low, and the binding of sub-micromolar cGMP to the regulatory GAF-B domain of the amino-terminus of PDE2 results in a 5-fold increase in cAMP hydrolysis rate (Martins et al., 1982; Martinez et al., 2002). The interplay between cAMP and cGMP signals through PDE2 has been well characterized 
mainly in the cardiovascular system (Maurice, 2005; Nikolaev et al., 2005), where cGMP-mediated regulation of cAMP occurs in a spatially confined cellular compartment and depends on the source of cGMP (Castro et al., 2006; Stangherlin et al., 2011). Regulation of cyclic nucleotides by PDE2 has already been shown in thalamic (Hepp et al., 2007) and striatal neurons (Lin et al., 2010), although in the striatum the role of PDE2 at the cellular level remained to be analyzed. Here, we improved the ${ }^{\mathrm{T}} \mathrm{Epac}^{V V}$ cAMP sensor (Klarenbeek et al., 2011) which allowed us to analyze the dynamics of cAMP regulation in striatal neurons and to determine the functional effect of cGMP on this signal.

Our data reveal that NO/cGMP signaling reduces the cAMP signals in both striatonigral and striatopallidal MSNs through the activation of PDE2. This inhibitory effect propagates downstream to PKA, leading to an inhibition of the PKA response to $\mathrm{D}_{1}$ stimulation. In addition, we found that the dynamics of the cAMP responses are not identical in MSNs, with striatopallidal neurons displaying larger and longer lasting cAMP transients than striatonigral MSNs.

\section{MATERIALS AND METHODS BIOSENSOR CONSTRUCT AND VALIDATION}

From the starting material TEpacVV (Klarenbeek et al., 2011), numbered Epac-SH74 in our database, we first exchanged mTurquoise for mTurqouise2 using the same protocol as in Klarenbeek et al., creating Epac-SH126. Second the Q270E mutation was introduced by cutting the Epac-SH126 with PshAI en BstEII inserting annealed oligo's forward primer: GTGACCCA TGGCAAGGGGCTGGTGACCACCCTGCATGAGGGAGATGAT TTTGGAGAGCTGGCTCTGGTCAATGATGCACCCCGGGCAG CCACCATCATCCTGCGAGAAGACAA and reverse primer: TTGTCTTCTCGCAGGATGATGGTGGCTGCCCGGGGTGCAT CATTGACCAGAGCCAGCTCTCCAAAATCATCTCCCTCATGC AGGGTGGTCACCAGCCCCTTGCCATGG, yielding EpacSH134. Third the acceptor was inserted as a PCR-product of cp174Citrine using forward primer: GGGGCTAGCGAGCTCAT GGACGGCGGCGTGCA and reverse primer: CGAATTCGG CTCGATGTTGTGGCGGAT digested with NheI and EcoRI, yielding Epac-SH150. All constructs were checked by sequence analysis. Hek293 embryonal kidney cells (American Type Culture Collection crl-1573) were cultured in DMEM supplemented with $10 \%$ FCS and antibiotics. Cells were seeded in six $15 \mathrm{~cm}^{2}$ plates and transfected with $10 \mu \mathrm{g}$ DNA per plate using calcium phosphate or fugene transfection agent. After overnight expression, cells were resuspended in $1 \mathrm{ml}$ hypotonic buffer (PBS: $\mathrm{H}_{2} \mathrm{O}$ 1: 2) and homogenized with a Downs piston. The homogenate was centrifuged for $10 \mathrm{~min}$ at $4^{\circ}$. The supernatant was corrected toward isotonic conditions using a concentrated stock of PBS. The supernatant was diluted 10 times in buffer containing (in mM) $150 \mathrm{KCl}, 5 \mathrm{NaCl}, 1 \mathrm{MgCl}_{2} 10$ HEPES pH 7.2, with a total volume of $2 \mathrm{ml}$ in a stirred cuvette of a PTI Quantamaster dual channel spectrofluorimeter (Lawrenceville, NJ). Small volumes of cAMP from concentrated stocks were added repeatedly to titrate in cAMP, total added volume $40 \mu$ l. The response to cAMP was quantified as the ratio between YFP $(530 \pm 10 \mathrm{~nm})$ and CFP $(490 \pm 10 \mathrm{~nm})$, when excited with $420 \pm 3 \mathrm{~nm}$.

\section{BRAIN SLICE PREPARATION}

Wild-type C57Bl/6J mice were obtained from Janvier (Le Genest Saint Isle, France). Mice were maintained in a $12 \mathrm{~h}$ light-12 h dark cycle, in stable conditions of temperature $\left(22^{\circ} \mathrm{C}\right)$, with food and water available ad libitum. All the experiments were performed according to French Ministry of Agriculture and Forestry guidelines for handling animals $\left(87-848^{\circ}\right)$.

Brain slices were prepared from male mice aged from 9 to 13 days, as previously described (Castro et al., 2013). Coronal brain slices of $300 \mu \mathrm{m}$ thickness were cut with a VT1200S microtome (Leica, Germany). Slices were prepared in an ice-cold solution of the following composition: $125 \mathrm{mM} \mathrm{NaCl}, 0.4 \mathrm{mM} \mathrm{CaCl}_{2}, 1 \mathrm{mM}$ $\mathrm{MgCl}_{2}, 1.25 \mathrm{mM} \mathrm{NaH}_{2} \mathrm{PO}_{4}, 26 \mathrm{mM} \mathrm{NaHCO} 3,25 \mathrm{mM}$ glucose and $1 \mathrm{mM}$ kynurenic acid, saturated with $5 \% \mathrm{CO}_{2}$ and $95 \%$ $\mathrm{O}_{2}$. The slices were incubated in this solution for $30 \mathrm{~min}$ and then placed on a Millicell-CM membrane (Millipore) in culture medium (50\% Minimum Essential Medium, 50\% Hanks' Balanced Salt Solution, $6.5 \mathrm{~g} / \mathrm{l}$ glucose, penicillin-streptomycin, Invitrogen). We used the Sindbis virus as a vector to induce expression of the various probes (Ehrengruber et al., 1999).

The sindbis viral vector for AKAR3 and Cygnet2 was prepared as as previously described (Gervasi et al., 2007; Hepp et al., 2007). Similarly, the Epac-S ${ }^{\mathrm{H} 150}$ digested with HindIII was inserted into pSinRep5 (Invitrogen, San Diego, CA) digested with StuI and made blunt by Klenow and HpaI.

Compared to our previous work (Castro et al., 2013), the viral vector was diluted to decrease the number of infected neurons and thus facilitate individual cell measurement. Slices were incubated overnight at $35^{\circ} \mathrm{C}$ under an atmosphere containing $5 \%$ $\mathrm{CO}_{2}$. Before the experiment, slices were incubated for $30 \mathrm{~min}$ in the recording solution (identical to the solution used for cutting, except that the calcium concentration was $2 \mathrm{mM}$ and kynurenic acid was omitted). During recordings, brain slices were continuously perfused with this solution saturated with $5 \% \mathrm{CO}_{2} / 95 \% \mathrm{O}_{2}$, at a rate of $2 \mathrm{ml} / \mathrm{min}$, in a recording chamber of $\sim 1 \mathrm{ml}$ volume maintained at $32^{\circ} \mathrm{C}$. The viability of the neurons in these experimental conditions have been checked by patch-clamp recording, which showed electrical activity to be normal (Gervasi et al., 2007; Castro et al., 2010).

\section{OPTICAL RECORDINGS ON BRAIN SLICES}

Recordings were made on MSNs, that constitute $95 \%$ of neurons in the striatum. Large neurons, presumably cholinergic interneurones, were excluded (i.e., diameter larger than $14 \mu \mathrm{m}$ ). Wide-field images were obtained with an Olympus BX50WI or BX51WI upright microscope with a $20 \times 0.5 \mathrm{NA}$ or a $40 \times$ 0.8 NA water-immersion objective and an ORCA-AG camera (Hamamatsu). Images were acquired with iVision (Biovision, Exton, PA, USA). The excitation and dichroic filters were D436/20 and $455 \mathrm{dcxt}$. Signals were acquired by alternating the emission filters, HQ480/40 for CFP, and D535/40 for YFP, with a filter wheel (Sutter Instruments, Novato, CA, USA). All filters were obtained from Chroma Technology (Brattleboro, VT, USA). Image acquisition was triggered manually, except for kinetics measurement where images were acquired automatically with $3-5 \mathrm{~s}$ intervals.

Images were analyzed with custom routines written in the IGOR Pro environment (Wavemetrics, Lake Oswego, OR, USA). 
The emission ratio was calculated for each pixel: F535/F480 for AKAR3 and F480/F535 for Epac-S ${ }^{\mathrm{H} 150}$. Pseudocolor images display the ratio value coded in hue and the fluorescence intensity coded in intensity. A calibration square indicates the intensity values from left to right and the ratio values from bottom to top. The size of the square indicates the scale of the image in microns. No correction for bleed-through or direct excitation of the acceptor was applied and the ratio changes in our conditions therefore appear smaller than those reported by other studies in which such corrections were applied.

\section{FAST DRUG APPLICATION}

A fast focal application system was previously used for kinetic studies (Gervasi et al., 2007; Castro et al., 2013). A glass pipette (100-150 $\mu \mathrm{m}$ tip diameter) was placed $300 \mu \mathrm{m}$ to the side of and $200 \mu \mathrm{m}$ above the brain slice and ejected the drug contained in the same solution as the bath. Dopamine uncaging was performed with UV light at $360 \mathrm{~nm}$ applied in wide-field mode (UVILED, Rapp OptoElectronic, Hamburg, Germany). Image acquisition in these fast wide-field recordings was automatic at a frequency ranging from 0.2 to $0.3 \mathrm{~Hz}$. Image acquisition was otherwise triggered manually by the user.

\section{DATA ANALYSIS AND STATISTICS}

Ratiometric quantification was performed with a ratio value between the Rmin and Rmax values, which correspond to the minimal ratio value (no biological signal) and maximal response (saturated biosensor) (Grynkiewicz et al., 1985; Börner et al., 2011). The baseline ratio in control conditions was considered to be equal to Rmin because adenylyl cyclase inhibition with $50 \mu \mathrm{M}$ SQ22536 and guanylyl cyclase inhibition with $10 \mu \mathrm{M}$ ODQ yielded no ratio decrease with Cygnet2 biosensor. The maximal response (Rmax, corresponding to biosensor saturation) was determined for each neuron at the end of the recording. This level was determined by applying $13 \mu \mathrm{M}$ forskolin (for cAMP) or SNAP (for cGMP) in the presence of the broad-spectrum phosphodiesterase inhibitor IBMX $(200 \mu \mathrm{M})$. Absolute ratio values differed between cells [as shown with the mutant biosensor in Castro et al. (2013)], so the amplitude of the response to receptor stimulation was quantified for each neuron as the fractional change in ratio from its own baseline (Rmin) and maximal final ratio response (Rmax).

Measurements were performed on regions of interest and some of the signal measured on a region of interest comes from out-of-focus neurons. Regions of interest which displayed clear responses to both SKF38393 and CGS21680 and which therefore contained fluorescence signal from out of focus cells were discarded from our analysis.

Kinetic parameters (amplitude, $t_{\max }$ and $t_{1 / 2 \text { off }}$ ) were determined using IGOR Pro environment (Wavemetrics, Lake Oswego, OR, USA). $t_{\max }$ values were determined as the time to reach the peak of the response and the $t_{1 / 2 \text { off represents the time to reach a }}$ half of the recovery of the response.

We analyzed at least four neurons per brain slice, with $n$ indicating the number of independent neurons tested. Unpaired two-tailed student's $t$-tests were used for statistical comparisons. Differences were considered significant when $P<0.001$.

\section{DRUGS}

SKF38393 hydrobromide, CGS21680 hydrochloride, 3-isobutyl1-methylxanthine (IBMX), rolipram, NPEC-caged dopamine [(N)-1-(2-nitrophenyl) ethylcarboxy-3, 4-dihydroxyphenethyl amine], and forskolin were obtained from Tocris Cookson (Bristol, UK); 1H-[1,2,4] oxadiazolo[4,3-a]quinoxalin-1-one (ODQ), S-nitroso-N-acetyl-D,L-penicillamine (SNAP), diethylamine NO (DEANO), erythro-9-(2-hydroxy-3-nonyl)-adenine (EHNA), BAY-60-7550 were obtained from Sigma-Aldrich (St Quentin Fallavier, France); BAY60-7550 was obtained from Cayman (Teaduspargi, Estonia).

\section{RESULTS}

\section{NO ACTIVATES THE SGC/cGMP SIGNALING CASCADE IN MSNs}

We used the cGMP sensor Cygnet2 (Honda et al., 2001) to determine whether NO donors increase cGMP concentration in MSNs. The NO donor S-nitroso-N-acetyl-D,L-penicillamine (SNAP, $100 \mu \mathrm{M}$ ) induced a large increase in the F480/F535 emission ratio of all the cygnet-expressing MSNs (Figure 1A). This signal reversed with the washout of the drug and a second cGMP response could be elicited from the same cells, showing that the NO-cGMP signaling pathway can be activated repeatedly over the time-course of our recordings.

The steady-state cGMP level upon sGC stimulation depends on the relative activities of $\mathrm{sGC}$ and cGMP degradation mediated by PDEs. In the presence of the NO donor, the non-specific PDE inhibitor IBMX $(200 \mu \mathrm{M})$ increased the ratio to a higher steady-state level, showing that PDE activities determine the steady-state cGMP level reached upon NO-mediated sGC stimulation. SNAP alone increased the emission ratio to $67 \pm 8 \%$ $(n=332)$ of this maximal response. Similar responses $(71 \pm$ $20 \% ; n=145)$ were obtained with the NO donor diethylamine NO (DEANO, $100 \mu \mathrm{M})$. As expected, sGC inhibition by ODQ $(10 \mu \mathrm{M})$ prevented the response to SNAP and SNAP plus IBMX (Figure 1B).

Previous studies performed with dissociated striatal neurons demonstrated that PDE2 regulates the CGMP responses to NO donors (Wykes et al., 2002; Lin et al., 2010). We tested the effect of PDE2 inhibition in our brain slice preparation and in all tested MSNs, the application of the PDE2 inhibitor EHNA $(10 \mu \mathrm{M})$, added on top of the SNAP response, induced a further increase of the cGMP signal, rising from $67 \pm 8 \%(n=332)$ to $90 \pm 5 \%$ $(n=151)$ of the maximal response obtained in the presence of IBMX (Figures 1C,D). Like IBMX, EHNA alone had no effect on basal cGMP levels.

These results confirm that PDE2 is critical in the regulation of the cGMP signals in MSNs of the dorsal striatum upon sGC stimulation.

\section{A NEW BIOSENSOR TO MEASURE cAMP SIGNALS IN THE STRIATUM}

Since cGMP increases PDE2 activity which also hydrolyzes cAMP, we wanted to precisely monitor whether PDE2 controlled cAMP levels in striatopallidal and striatonigral MSN. The recently published ${ }^{\mathrm{T}} \mathrm{Epac}^{\mathrm{VV}}$ (Epac-S $\left.{ }^{\mathrm{H} 126}\right)$ exhibits one of the largest ratio changes known to date for a genetically-encoded biosensors (Klarenbeek et al., 2011) but we considered that its relatively low sensitivity for cAMP may be limiting. We have prepared a 


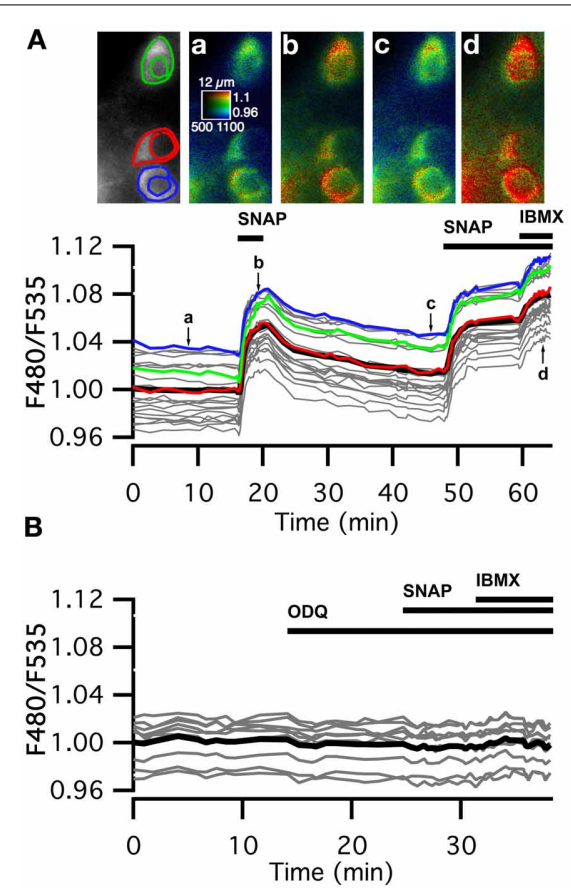

FIGURE 1 | Cygnet reports increases in cyclic GMP concentration in all medium spiny neurons of the striatum in response to soluble guanylyl cyclase activation. (A-C) Striatal neurons in a mouse brain slice expressing Cygnet2 sensor and imaged by wide-field microscopy. (A) Images show the raw fluorescence at $535 \mathrm{~nm}$ (left in gray scale) and the ratio (in pseudocolor), indicating the ratiometric change of Cygnet2 reporting the binding of cGMP, at the times indicated by the corresponding arrows on the graph below. Each trace on the graph indicates the F480/F535 emission ratio measurement on regions indicated by the color contour drawn on the raw image; the thick black line represents the average of all the traces. The NO donor SNAP

$(100 \mu \mathrm{M})$ induced a strong increase in the ratio in all MSNs visible in the field.
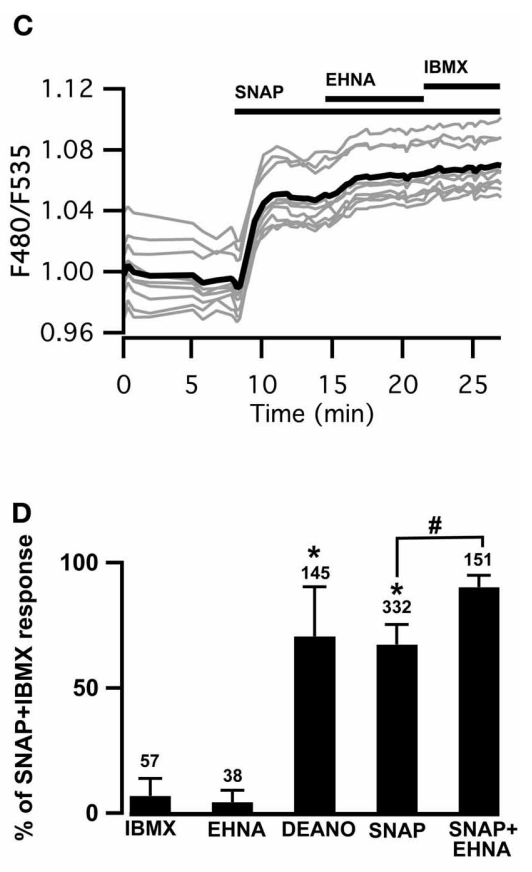

SNAP + IBMX $(200 \mu \mathrm{M})$ induced a maximal increase in the ratio response. (B) The responses to SNAP and SNAP + IBMX were completely blocked by the soluble guanylyl cyclase blocker ODQ $(10 \mu \mathrm{M})$. (C) After the activation of SGC with SNAP, the PDE2 inhibitor EHNA $(10 \mu \mathrm{M})$ increased the cGMP signal to a level that corresponded to the maximal response. (D) The amplitude measured with the Cygnet2 biosensor was normalized with respect to the maximal SNAP + IBMX response and plotted as a histogram. Error bars indicate s.e.m.; the numbers of tested neurons are indicated above each bar. Unpaired two-tailed $t$-tests were carried out for comparisons with control conditions $\left(^{*}\right)$ and with SNAP alone (\#), and differences were considered significant when $P<0.05$. series of new Epac-based cAMP biosensors in which the donor was replaced by mTurquoise2, which has a higher quantum yield, longer lifetime and is more photo stable than mTurquoise (Goedhart et al., 2012). The Q270E mutation (Dao et al., 2006) was introduced in the cAMP binding site of the cAMP-binding domain of Epac1 to increase its affinity for cAMP. This sensor, called Epac-S $\mathrm{H}^{\mathrm{H} 134}$ showed increased sensitivity to cAMP as compared to the previous version $\left(\mathrm{EC}_{50}\right.$ was $4.4 \pm 0.3$ vs. $10.7 \pm$ $0.8 \mu \mathrm{M}, n=5, p<0.01$, two-tailed paired $t$-test; Figure $2 \mathrm{~A}$ ). This sensor was further improved by replacing the acceptor cpVenus-Venus with a single circular permutation of Citrine (cp174Citrine), chosen for optimal resistance to $\mathrm{pH}$ changes and brightness. This sensor called Epac-S ${ }^{\mathrm{H} 150}$ showed a large change in emission spectrum upon cAMP binding (Figure 2B) and proved suitable to directly address the dynamics of cAMP in MSNs in the striatum (Figure 2C). A detailed characterization of this and other new CAMP sensors will be published elsewhere.

As shown previously with PKA and cAMP biosensors with two-photon microscopy (Castro et al., 2013), activation of the $\mathrm{D}_{1}$ receptors with a saturating $(1 \mu \mathrm{M})$ dose of SKF38393 strongly increased the F480/F535 emission ratio in one population of MSNs called hereafter $D_{1}$ neurons. In addition, the activation of the $A_{2 A}$ receptors with a saturating $(1 \mu \mathrm{M})$ dose of CGS21680 increased the emission ratio in the remaining neuronal population, the $\mathrm{D}_{2}$ neurons (Figure 2C). With low viral infection levels, wide-field imaging thus allowed a sufficient cell separation to distinguish between the $D_{1}$ and $D_{2}$ neurons. The $D_{1}$ response decreased with prolonged exposure to the agonist, probably a consequence of $D_{1}$ receptor desensitization. In contrast, the response to CGS21680 reached a stable steady-state level. Both responses were not maximal, as the addition of the adenylyl cyclase activator forskolin (FSK, $13 \mu \mathrm{M})$ further increased the ratio response, consistent with the wide cAMP sensitivity range of this new biosensor. Addition of $200 \mu \mathrm{M}$ IBMX to forskolin produced a small additional response, considered as the maximal ratio response $R_{\max }$ (see methods). On average, SKF38393, CGS21680, and FSK increased the emission ration to $51 \pm 2 \%$ $(n=32), 27 \pm 1 \%(n=29)$ and $95 \pm 1 \%(n=48)$ of the maximal response to forskolin and IBMX (Figure 2D).

\section{BRIEF ADENYLYL CYCLASE ACTIVATION IN $D_{\mathbf{1}}$ AND $D_{\mathbf{2}}$ NEURONS}

This imaging method thus allowed us to analyze the cAMP signal in identified $\mathrm{D}_{1}$ or $\mathrm{D}_{2}$ MSNs and we set up a protocol for transient adenylyl cyclase stimulation to analyze the onset, which mostly reflect cAMP synthesis, and the decay, which is mostly 


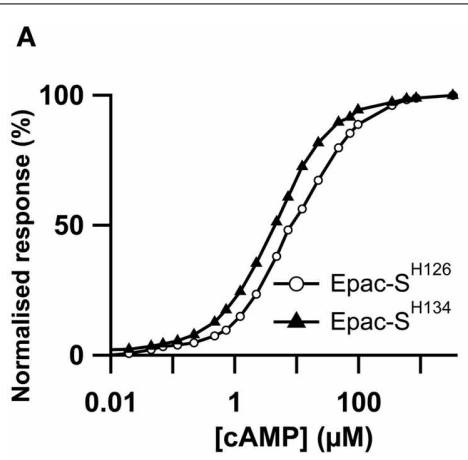

C
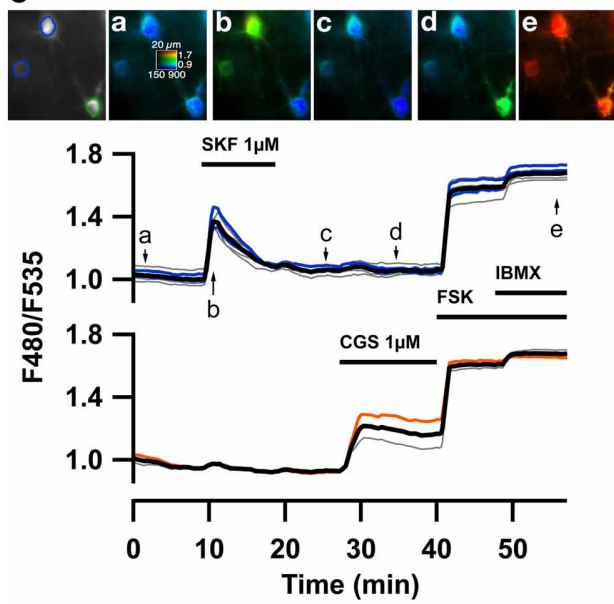

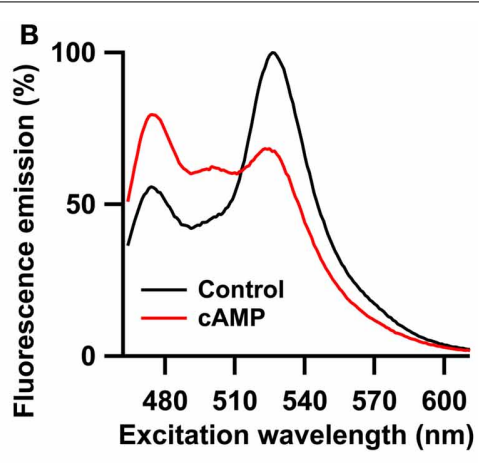

D

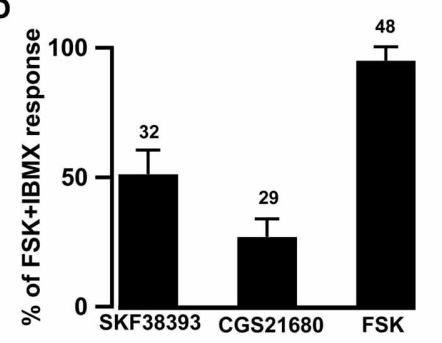

FIGURE 2 | The Epac-S ${ }^{\mathrm{H} 150}$ sensor reported large cAMP signals in $\mathrm{D}_{\mathbf{1}}$ and $\mathbf{D}_{\mathbf{2}}$ MSNs. The ${ }^{\mathrm{T}}$ Epac ${ }^{\mathrm{V}}$ sensor (labeled Epac- $\mathrm{S}^{\mathrm{H} 126}$ ) was mutated to increase its sensitivity for CAMP, yielding Epac- $\mathrm{S}^{\mathrm{H} 134}$; representative dose-response curves for both sensors are presented in (A). The acceptor was then changed for a cp174Citrine, yielding Epac- $\mathrm{S}^{\mathrm{H} 150}$. The emission spectrum of Epac-S $\mathrm{S}^{\mathrm{H150}}$ in the absence and in the presence of saturating $(100 \mu \mathrm{M})$ cAMP is presented in (B). (C) Medium spiny neurons (MSNs) in a mouse brain slice were transfected for expression of Epac-S ${ }^{\mathrm{H} 150}$ and imaged with wide field microscopy. Images show the raw fluorescence at $535 \mathrm{~nm}$ (left, in gray scale) and the ratio (in pseudocolor) indicating the ratiometric change of Epac-S ${ }^{H 150}$ reporting the binding of CAMP, at the times indicated by the arrows on the graph below. Each trace on the graph indicates the F480/F535 emission ratio measurement on regions indicated by the color contour drawn on the raw image; the thick black line represents the average of all the traces in the two different groups. The $D_{1}$ receptor activator, SKF38393 $(1 \mu \mathrm{M})$, induced a transient ratio increase in neurons thereby identified as $D_{1} M S N s$; activation of the $A_{2 A}$ receptor with CGS21680 $(1 \mu \mathrm{M})$ induced a sustained cAMP response in neurons thereby identified as $D_{2}$ MSNs. FSK + IBMX induced a ratio increase used as $100 \%$ value in normalization. (D) The amplitude of the responses to SKF38393 and CGS21680 was measured with the Epac-S ${ }^{\mathrm{H} 150}$ biosensor, normalized with respect to the maximal FSK + IBMX response and plotted as a histogram. The cAMP response to $D_{1}$ receptor stimulation (SKF38393) was significantly stronger than the response to $D_{2}$ receptor stimulation. Error bars indicate the s.e.m.; the number of tested neurons is indicated above each bar. governed by PDEs activities. Because the activation of $\mathrm{D}_{1}$ or $\mathrm{A}_{2} \mathrm{~A}$ receptors induced cAMP signals that differed in amplitude and kinetics, we examined the responses to direct stimulation of AC by forskolin, thereby analyzing the cAMP signal independently of receptors. We used a fast focal application system to apply 10-s pulses of forskolin (FSK) while monitoring the cAMP response, and bath application of SKF38393 and CGS21680 at the end of the recording to identify $\mathrm{D}_{1}$ and $\mathrm{D}_{2}$ neurons (Figure $3 \mathrm{~A}$ ). For both $\mathrm{D}_{1}$ and $\mathrm{D}_{2}$ MSNs, brief forskolin stimulation resulted in a transient increase in Epac- $\mathrm{S}^{\mathrm{H150}}$ signal, which could be reproduced several times with no significant change in amplitude or kinetics. These forskolin-induced cAMP transients differed between the two types of MSNs in their amplitude and decay kinetics (Figure 3B): $\mathrm{D}_{1}$ MSNs generated a cAMP transient that reached

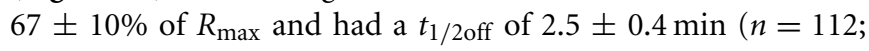

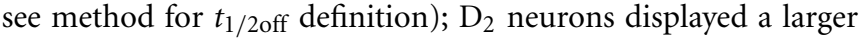
cAMP signal that reached $79 \pm 9 \%$ of $R_{\max }$ and lasted two times longer than the response in $\mathrm{D}_{1}$ neurons with a $t_{1 / 2 \text { off }}$ of $4.3 \pm$ $0.8 \mathrm{~min}(n=122)$; both amplitude and $t_{1 / 2 \text { off }}$ were statistically different from $\mathrm{D}_{1}$ (unpaired two-tailed $t$-test, $P<0.0001$; see Table 1).

We thought that this difference might result from regulation of cAMP levels by PDEs, including PDE2. We observed that inhibition of PDE2 with EHNA increased the amplitude and prolonged the decay of the forskolin-induced cAMP transients exclusively in $\mathrm{D}_{2} \mathrm{MSNs}$ (Figure $3 \mathrm{C}$ ). No effect was observed in $\mathrm{D}_{1} \mathrm{MSN}$, despite the expression of PDE2 in these neurons (Figure 3D) (Lin et al., 2010). Because PDE2 inhibition exacerbates the differences between $D_{1}$ and $D_{2}$ MSNs, we hypothesized that other PDE activities differently regulate the cAMP signals in these neurons. We 

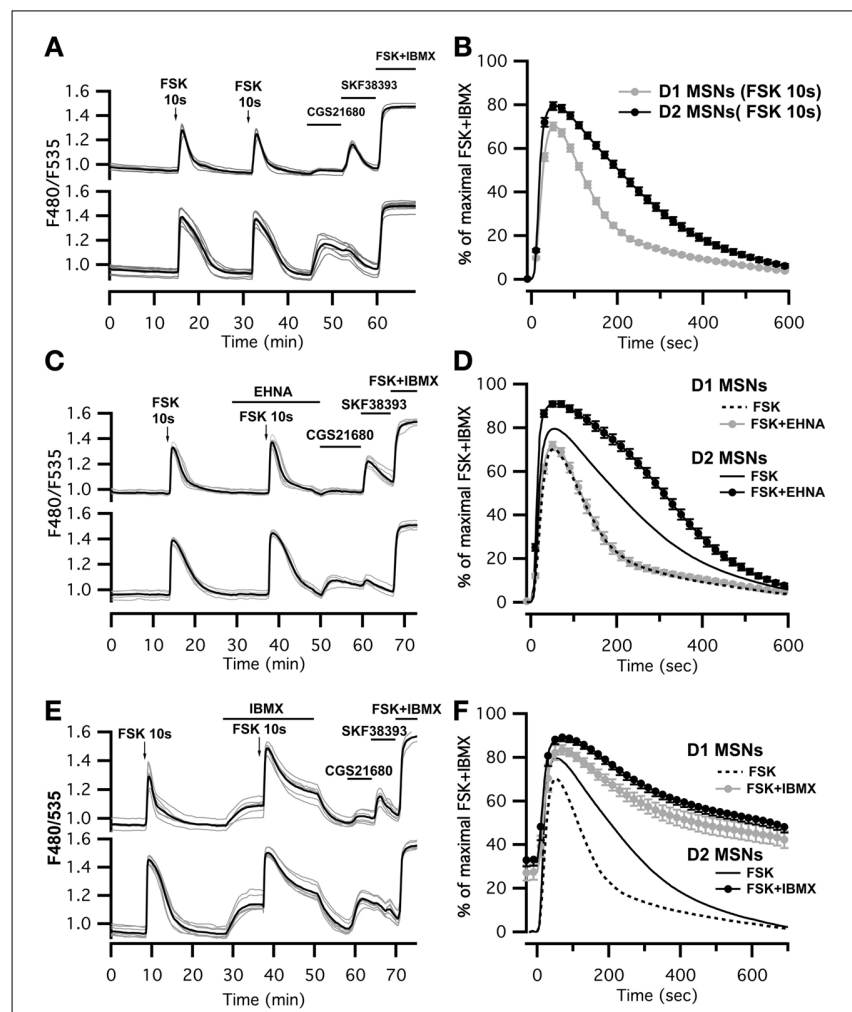

FIGURE 3 | Brief (10 s) application of FSK (13 $\mu \mathrm{M})$ produced stronger and longer lasting cAMP responses in $D_{2}$ MSNs than in $D_{1}$ MSNs. $(\mathbf{A}, \mathbf{C}, \mathbf{E})$ CAMP signals were measured in wide-field imaging with Epac-S $\mathrm{S}^{\mathrm{1} 150}$ biosensor during the application of a $10 \mathrm{~s}$ pulse of FSK alone (A), in the presence of EHNA (C) or IBMX (E). Each trace on the graph indicate the Epac-S ${ }^{\mathrm{H} 150}$ emission ratio of an individual neuron. Traces were separated on the basis of their response to the $A_{2 A}$ agonist CGS21680 $(1 \mu \mathrm{M})$ or $D_{1}$ agonist SKF38393 $(1 \mu \mathrm{M})$. (B) Mean responses in $D_{1}(n=112)$ and $D_{2}(n=122)$ MSNs to brief FSK stimulation. (D,F) Mean responses in $D_{1}$ and $D_{2}$ to brief FSK stimulation in the presence of EHNA $(10 \mu \mathrm{M})$ or IBMX $(200 \mu \mathrm{M})$. Thin dashed and solid lines represent the mean responses in $D_{1}$ and $D_{2}$ MSNs to brief FSK alone, for comparison. IBMX strongly attenuated the differences between $D_{1}(n=17)$ and $D_{2}(n=21)$ MSNs. EHNA increased the cAMP signaling in $D_{2}$ MSNs $(n=19)$, but had no effect in $D_{1}$ cells $(n=19)$. Markers are plotted sparsely for clarity; error bars represent $S D$. tested this hypothesis by inhibiting most of PDE activities with IBMX (Figures 3E,F). Application of IBMX alone increased basal cAMP levels in both type of cells $[28 \pm 3 \%(n=17)$ for D1 MSNs and $33 \pm 3 \%(n=21)$ for D2 MSNs], showing that adenylyl cyclase constitutively active. When most PDEs were blocked, the cAMP transient induced with $10 \mathrm{~s}$ FSK was larger in amplitude and its duration was considerably prolonged. The difference in kinetics between $\mathrm{D}_{1}$ and $\mathrm{D}_{2}$ was almost obliterated, demonstrating that phosphodiesterase activities differ between $\mathrm{D}_{1}$ and $\mathrm{D}_{2}$ neurons. The slope of the $10-80 \%$ onset of the cAMP transient in the presence of IBMX was measured and showed no statistical difference between $\mathrm{D}_{1}$ and $\mathrm{D}_{2}$ neurons $(0.020 \pm 0.005$, $n=17$ for $\mathrm{D}_{1}$ MSNs vs. $0.023 \pm 0.007, n=20$ for $\mathrm{D}_{2}$ MSNs, expressed in ratio units per second, $p=0.199$ ). This suggests that the rate of cAMP synthesis is similar in both cell types. Further work is needed to identify the specific contribution of each PDEs in determining the shape of transient cAMP signals in $\mathrm{D}_{1}$ and $\mathrm{D}_{2}$ MSNs.

\section{PDE2 ACTIVATION BY cGMP LIMITS cAMP ACCUMULATION IN BOTH $D_{1}$ AND $D_{2}$ MSNs}

We then tested whether increasing PDE2 activity with cGMP affected cAMP signaling in MSNs: we compared the cAMP transients in the absence and in the presence of the NO donor SNAP $(100 \mu \mathrm{M})$. Application of SNAP in the bath had no effect per se on basal cAMP signals (Figure 4A). However, the cAMP transient was reduced in amplitude by about $20-30 \%$ in both striatonigral and striatopallidal MSNs (Figure 4B; Table 1). The decay timecourse, however, did not change significantly from the respective control (Figure 4B; Table 1).

This negative control exerted by $\mathrm{NO}$ was abolished when the PDE2 inhibitor EHNA $(10 \mu \mathrm{M})$ was applied simultaneously with the NO donor SNAP $(100 \mu \mathrm{M})$ (Figures 4C,D; Table 1). The application of EHNA alone or in the presence of SNAP had no effect on basal cAMP signal (Figure 4C). Since EHNA also inhibits adenosine deaminase, we also tested the specific PDE2 inhibitor BAY60-7550 at $100 \mathrm{nM}$ (Figures 4E,F): this drug also blocked the inhibitory effect of NO donors on cAMP transients. These results show that the inhibitory crosstalk exerted by cGMP on cAMP transients is mediated by PDE2.

Table 1 | Kinetic parameters of the cAMP responses to FSK in MSNs.

\begin{tabular}{|c|c|c|c|c|c|c|c|c|}
\hline & \multicolumn{4}{|c|}{ D1 } & \multicolumn{4}{|c|}{ D2 } \\
\hline & Amplitude (\%) & $t_{1 / 2 \text { off }}(\min )$ & $t_{\max }(s)$ & $n$ & Amplitude (\%) & $t_{1 / 2 \text { off }}(\min )$ & $t_{\max }(s)$ & $n$ \\
\hline FSK & $67 \pm 10$ & $2.6 \pm 0.4$ & $52 \pm 10$ & 112 & $79 \pm 9$ & $4.3 \pm 0.8$ & $51 \pm 8$ & 122 \\
\hline FSK + SNAP & $41 \pm 8$ & $2.9 \pm 0.6$ & $54 \pm 9$ & 45 & $62 \pm 7$ & $4.0 \pm 0.5$ & $53 \pm 7$ & 48 \\
\hline $\mathrm{FSK}+\mathrm{EHNA}$ & $72 \pm 8$ & $2.5 \pm 0.6$ & $49 \pm 7$ & 19 & $91 \pm 5$ & $5.4 \pm 0.8$ & $55 \pm 9$ & 19 \\
\hline FSK + SNAP + EHNA & $66 \pm 9$ & $2.6 \pm 0.7$ & $58 \pm 13$ & 46 & $84 \pm 6$ & $5.2 \pm 1.1$ & $54 \pm 7$ & 44 \\
\hline FSK + SNAP + Bay 607,550 & $60 \pm 12$ & $2.0 \pm 0.3$ & $46 \pm 10$ & 38 & $84 \pm 12$ & $5.3 \pm 2.2$ & $59 \pm 9$ & 25 \\
\hline
\end{tabular}

CAMP signals were measure in wide-field imaging with Epac-S ${ }^{H 150}$ biosensor during the application of a $10 \mathrm{~s}$ pulse of Forskoline (FSK, $\left.13 \mu \mathrm{M}\right)$ alone or in the presence of SNAP $(100 \mu \mathrm{M})$, EHNA $(10 \mu \mathrm{M}), S N A P+E H N A$ and SNAP + Bay 607,550. The table shows the means $( \pm S D)$ of the amplitudes, the $t_{\text {max }}$ and $t_{1 / 2}$ off in each experimental condition. $n$ indicate the number of tested neurons. 


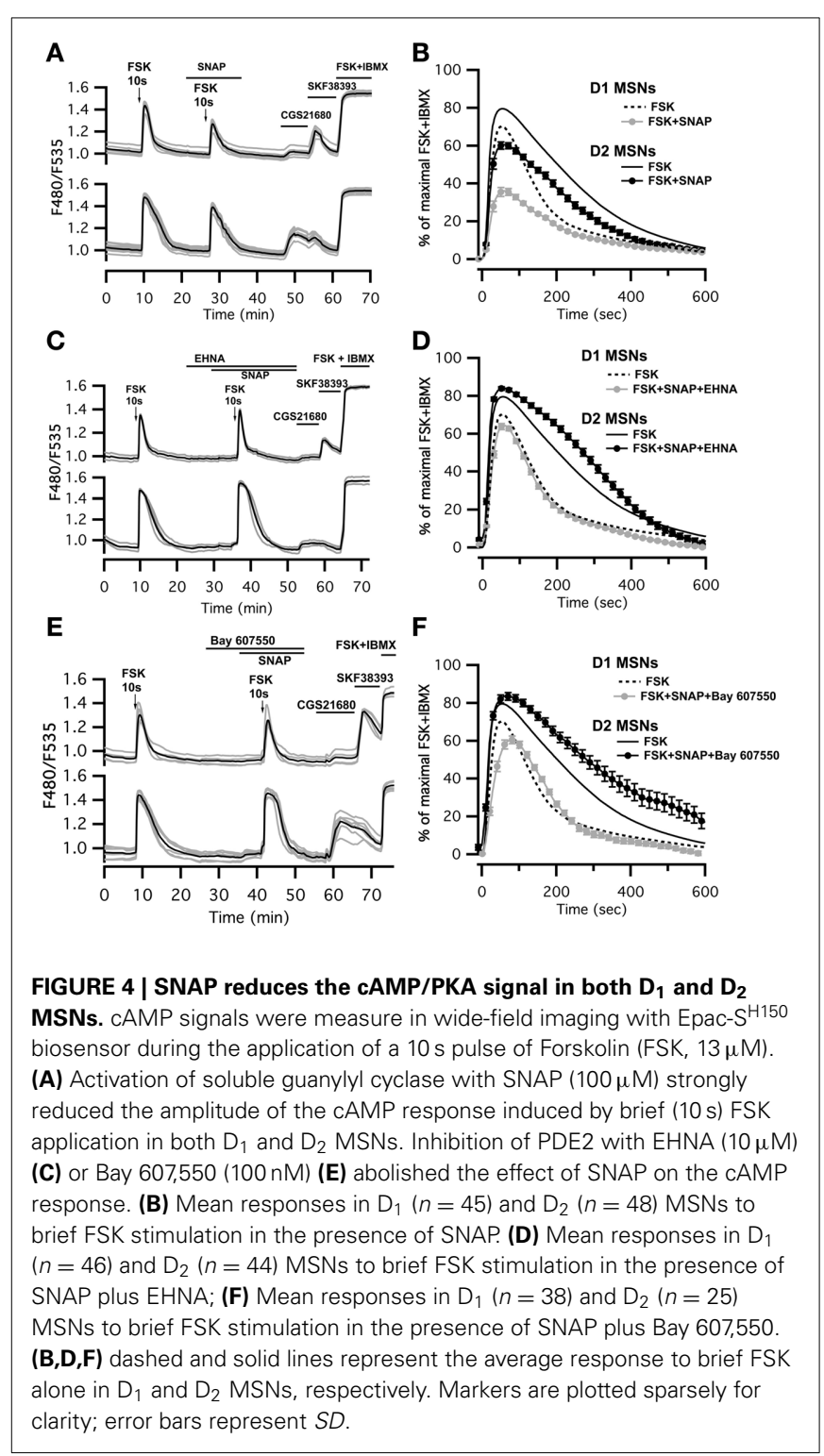

\section{THE NO/cGMP/PDE2 PATHWAY LIMITS PKA ACTIVATION UPON A SUB-SECOND DOPAMINE SIGNAL}

Finally, we checked whether the negative regulation exerted by cGMP on the cAMP signal was sufficient to down-regulate PKA activity. Physiological activation of $\mathrm{D}_{1}$-like receptors is associated with phasic dopamine release related to a reward or novelty (Garris and Wightman, 1994; Gonon, 1997; Schultz and Dickinson, 2000). We mimicked phasic dopamine signals by uncaging NPEC-dopamine with a flash of UV light of $1 \mathrm{~s}$ duration while monitoring PKA activity with AKAR3. At the end of the experiment, bath application of SKF38393 allowed for the identification of $D_{1}$ neurons (Figure 5A). $D_{2}$ neurons, which did not respond to dopamine uncaging or SKF38393, were ignored. As already described (Castro et al., 2013), $\mathrm{D}_{1}$ MSNs displayed a large and transient response to $1 \mathrm{~s}$ dopamine uncaging that reached $90 \pm 2 \% n=84$ of the maximal steadystate response to SKF38393 response (Figure 5B). In the presence

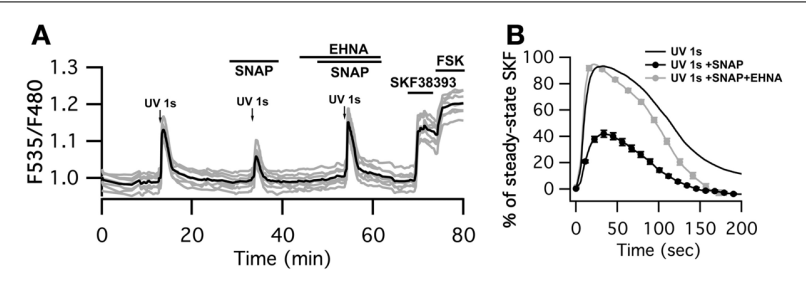

FIGURE 5 | SNAP inhibits the PKA response to brief (1 s) dopamine uncaging in $\mathbf{D}_{\mathbf{1}}$ MSNs. (A) MSNs expressing AKAR3 were imaged in wide-field microscopy to measure the PKA response to dopamine. Arrows indicate the UV flash. Flash photolysis of caged dopamine (NPEC-dopamine, $5 \mu \mathrm{M}$ in the bath) induced a transient ratio increase. SNAP decreased this response, and the PDE2 inhibitor EHNA $(10 \mu \mathrm{M})$ restored the response to caged dopamine to its initial level. (B) Mean responses in $D_{1}$ to $1 \mathrm{~s}$ UV flash alone $(n=84)$, in the presence of SNAP $(n=80)$ and SNAP + EHNA $(n=74)$. For each flash, responses were normalized with respect to the steady-state response to SKF38393. Markers are plotted sparsely for clarity; error bars represent $S D$.

of the NO donor SNAP, the amplitude of the PKA signal was decreased by $45 \%(42 \pm 3 \% n=80)$. PDE2 inhibition by $10 \mu \mathrm{M}$ EHNA reverted the inhibitory effect of SNAP $(93 \pm 4 \% n=74)$ showing that PDE2 is indeed the mediator of this inhibitory effect.

Interestingly, the inhibitory effect of SNAP appeared larger at the level of PKA than at the level of cAMP, showing that a moderate change in cAMP dynamics is amplified at the PKA level.

\section{DISCUSSION}

Previous studies have clearly highlighted NO/cGMP as a key player in detecting and reinforcing corticostriatal glutamatergic and dopaminergic neurotransmission (Calabresi et al., 2007; West and Tseng, 2011). In this report, we highlight the importance of the post-synaptic level where NO affects the integrative properties of MSNs. Our data demonstrate the functional importance of PDE2 as a target of the NO/cGMP pathway, and its importance in the regulation of transient cAMP responses. With the high resolution provided by our improved biosensor, we also highlight significant differences between $D_{1}$ and $D_{2}$ MSNs in their response to transient $\mathrm{AC}$ stimulation, a difference that results from differences in phosphodiesterase activities.

\section{PDE2 AS A MAJOR TARGET OF cGMP IN THE STRIATUM}

The physiological effects of the NO/cGMP pathway largely depend on its targets which include cGMP-gated ion channels, cGMP-dependent kinase protein (PKG) and cGMP-regulated PDEs. Interestingly, brain regions such as the hippocampus and the striatum which express high levels of sGC (Matsuoka et al., 1992; Ding et al., 2004), do not express much PKG protein (elHusseini et al., 1995; El-Husseini et al., 1999) but very high levels of PDE2 (Repaske et al., 1993; Van Staveren et al., 2003). This suggests that the major role of cGMP may be to regulate cAMP levels via PDE2 activation, rather than to directly activate PKG. This hypothesis is clearly supported by our experiments which show that one major functional effect of NO-triggered cGMP production is the activation of PDE2 which then strongly reduce the cAMP response in both $\mathrm{D}_{1}$ and $\mathrm{D}_{2}$ neurons. 
The inhibitory effect of $\mathrm{NO}$ on the transient response was much more pronounced when considering the effect at the PKA level than at the cAMP level. This results appears counterintuitive as one might expect PKA to smooth-out small variations in cAMP concentration. This non-linearity in signal transduction from cAMP level to PKA level may result from positive feedback controls such as that mediated by DARPP-32: DARPP-32 was shown to prolong the PKA response once it was activated (Le Novere et al., 2008; Castro et al., 2013); in our experiments, a cAMP response that has been lowered by the NO-cGMP pathway may be insufficient to phosphorylate DARPP-32, while a larger cAMP signal may phosphorylate more DARPP-32, therefore inhibit Phosphatase 1 more efficiently and thus potentiate the net PKA effect. Such non-linear effects certainly determine the integrative properties of striatal neurons and further modeling studies are needed to understand how this parameter comes into play in the current learning theories.

\section{DICHOTOMOUS CAMP RESPONSES IN MSNS}

While the characteristic segregation of MSNs in two different cell types is notoriously difficult to study on dissociated neurons, biosensor imaging provides a measurement at the level of individual mature neurons which can be identified on the basis of their response to an agonist of either dopamine $\mathrm{D}_{1}$ or adenosine $A_{2 A}$ receptor (Castro et al., 2013). In this study, the ${ }^{T} \mathrm{Epac}^{\mathrm{VV}}$ cAMP sensor was improved to increase its affinity for cAMP and decrease it size, yielding a biosensor which proved highly efficient to monitor cAMP signals in striatal neurons. This new biosensor revealed the larger and desensitizing profile of the $D_{1}$ response which differed from the smaller and steady $A_{2 A}$ response in $D_{2}$ MSNs. These differences may be related to different regulations at the level of receptor transduction and remain to be explored in more details.

Our experiments also revealed a clear differences between these two types of MSNs that lie downstream of the receptors, which appeared when AC was transiently stimulated with forskolin: the cAMP signals were stronger and lasted longer in D2 MSNs than in D1 neurons. The AC activities do not explain the differences observed in these cells because the rate of cAMP production upon forskolin stimulation was similar in D1 and D2 MSNs as indicated by a similar onset slope. This dichotomy depends on differences in PDE activity since the broad-spectrum PDE inhibitor, IBMX, suppressed the differences between MSNs. Specific inhibition of PDE2 increased the cAMP signal exclusively in D2 neurons, despite the expression of PDE2 in both D1 and D2 neurons (Lin et al., 2010): this suggests that PDE2 is at the forefront in the degradation of cAMP in D2 neurons. Transient cAMP responses recover faster in D1 neurons than in D2 neurons, and this recovery is not affected by PDE2 inhibition suggesting that another PDE takes over PDE2 in D1 neurons. Based on these results, we consider that amplitude and decay of the cAMP signal are governed by a complex and non-linear network, which may include subcellular compartmentation, in which PDE2 appears as one critical element. Which PDE (or combination) is at the forefront in D1 neurons remains to be determined, possibly PDE1A (Polli and Kincaid, 1994) and/or PDE10A (Xie et al., 2006).

\section{NO INCREASES cGMP LEVELS IN BOTH TYPES OF MSNs}

This clear-cut segregation of MSNs into two populations with different pharmacological profiles and PDE activities that was observed for the cAMP/PKA signaling cascade was not seen at the level of cGMP with the cGMP biosensor Cygnet: sGC stimulation with NO donors produced a large and homogeneous increase in cGMP levels in all MSNs, which is consistent with the high levels of sGC present in both neuron types (Ding et al., 2004).

A tonic production of cGMP in response to the spontaneous release of NO was reported in the hippocampus, optic nerve, thalamus (Garthwaite et al., 2006; Hopper and Garthwaite, 2006; Hepp et al., 2007), as well as in the striatum (Calabresi et al., 1999; Galati et al., 2008). Our experiments in MSNs contrast with these observations as no changes in cGMP concentration was obtained upon bath application of ODQ or IBMX (Figure 1). However, data in the striatum were obtained in quite different experimental conditions: either the glutamatergic corticostriatal afferent fibers were repeatedly stimulated which should activate NOS interneurons (Calabresi et al., 1999), or the recordings were performed in vivo where a number of other parameters can directly or indirectly contribute to a tonic activation of these NO-synthesizing cells (West and Grace, 2000, 2004; Galati et al., 2008). This suggests that, in vivo, NOergic interneurons tonically release NO which downregulate the dopamine-induced PKA response of MSNs. Integrating this cellular mechanism into the whole animal would be of paramount importance to elucidate the clinical influence of compounds interfering with NO/cGMP pathway in disabling disorders associated with the striatum.

\section{AUTHOR CONTRIBUTIONS}

Liliana R. V. Castro and Pierre Vincent contributed equally to this work (co-last authors). Danièle Paupardin-Tritsch, Kees Jalink, Pierre Vincent, and Liliana R. V. Castro supervised the project. Liliana R. V. Castro, Marina Polito, and Jeffrey Klarenbeek performed the experiments. Liliana R. V. Castro, Marina Polito, Danièle Paupardin-Tritsch, and Pierre Vincent wrote the manuscript.

\section{ACKNOWLEDGMENTS}

This work was supported by Centre National de la Recherche Scientifique. The team of Marina Polito, Danièle PaupardinTritsch, Pierre Vincent, and Liliana R. V. Castro is part of the École des Neurosciences de Paris Ile-de-France and Labex BioPsy networks. We thank Regine Hepp for producing the sindbis virus. Initial characterization of novel Epac sensors was carried out during a visit of Pierre Vincent and Marina Polito to the Jalink lab in the context of the Proof-of-Concept phase of EuroBioImaging.

\section{REFERENCES}

Ariano, M. A., Lewicki, J. A., Brandwein, H. J., and Murad, F. (1982) Immunohistochemical localization of guanylate cyclase within neurons of rat brain. Proc. Natl. Acad. Sci. U.S.A. 79, 1316-1320. doi: 10.1073/pnas.79.4.1316

Bateup, H. S., Svenningsson, P., Kuroiwa, M., Gong, S., Nishi, A., Heintz, N., et al. (2008). Cell type-specific regulation of DARPP-32 phosphorylation by psychostimulant and antipsychotic drugs. Nat. Neurosci. 11, 932-939. doi: $10.1038 / \mathrm{nn} .2153$ 
Bertran-Gonzalez, J., Bosch, C., Maroteaux, M., Matamales, M., Herve, D., Valjent, E., et al. (2008). Opposing patterns of signaling activation in dopamine D1 and D2 receptor-expressing striatal neurons in response to cocaine and haloperidol. J. Neurosci. 28, 5671-5685. doi: 10.1523/JNEUROSCI.103908.2008

Börner, S., Schwede, F., Schlipp, A., Berisha, F., Calebiro, D., Lohse, M. J., et al. (2011). FRET measurements of intracellular cAMP concentrations and cAMP analog permeability in intact cells. Nat. Protoc. 6, 427-438. doi: 10.1038/nprot.2010.198

Calabresi, P., Centonze, D., Gubellini, P., Marfia, G. A., Pisani, A., Sancesario, G., et al. (2000). Synaptic transmission in the striatum: from plasticity to neurodegeneration. Prog. Neurobiol. 61, 231-265. doi: 10.1016/S0301-0082(99) 00030-1

Calabresi, P., Gubellini, P., Centonze, D., Sancesario, G., Morello, M., Giorgi, M., et al. (1999). A critical role of the nitric oxide/cGMP pathway in corticostriatal long-term depression. J. Neurosci. 19, 2489-2499.

Calabresi, P., Picconi, B., Tozzi, A., and Di Filippo, M. (2007). Dopamine-mediated regulation of corticostriatal synaptic plasticity. Trends Neurosci. 30, 211-219. doi: 10.1016/j.tins.2007.03.001

Castro, L. R., Brito, M., Guiot, E., Polito, M., Korn, C. W., Herve, D., et al. (2013). Striatal neurones have a specific ability to respond to phasic dopamine release. J. Physiol. 591, 3197-3214. doi: 10.1113/jphysiol.2013.252197

Castro, L. R., Gervasi, N., Guiot, E., Cavellini, L., Nikolaev, V. O., PaupardinTritsch, D., et al. (2010). Type 4 phosphodiesterase plays different integrating roles in different cellular domains in pyramidal cortical neurons. J. Neurosci. 30, 6143-6151. doi: 10.1523/JNEUROSCI.5851-09.2010

Castro, L. R., Verde, I., Cooper, D. M., and Fischmeister, R. (2006). Cyclic guanosine monophosphate compartmentation in rat cardiac myocytes. Circulation 113, 2221-2228. doi: 10.1161/CIRCULATIONAHA.105.599241

Dao, K. K., Teigen, K., Kopperud, R., Hodneland, E., Schwede, F., Christensen, A. E., et al. (2006). Epacl and cAMP-dependent protein kinase holoenzyme have similar cAMP affinity, but their cAMP domains have distinct structural features and cyclic nucleotide recognition. J. Biol. Chem. 281, 21500-21511. doi: 10.1074/jbc.M603116200

de Vente, J., Asan, E., Gambaryan, S., Markerink-van Ittersum, M., Axer, H., Gallatz, K., et al. (2001). Localization of cGMP-dependent protein kinase type II in rat brain. Neuroscience 108, 27-49. doi: 10.1016/S0306-4522(01) 00401-8

Ding, J. D., Burette, A., Nedvetsky, P. I., Schmidt, H. H., and Weinberg, R. J. (2004). Distribution of soluble guanylyl cyclase in the rat brain. J. Comp. Neurol. 472, 437-448. doi: 10.1002/cne.20054

Ehrengruber, M. U., Lundstrom, K., Schweitzer, C., Heuss, C., Schlesinger, S., and Gahwiler, B. H. (1999). Recombinant Semliki Forest virus and Sindbis virus efficiently infect neurons in hippocampal slice cultures. Proc. Natl. Acad. Sci. U.S.A. 96, 7041-7046. doi: 10.1073/pnas.96.12.7041

el-Husseini, A. E., Bladen, C., and Vincent, S. R. (1995). Molecular characterization of a type II cyclic GMP-dependent protein kinase expressed in the rat brain. J. Neurochem. 64, 2814-2817. doi: 10.1046/j.1471-4159.1995. 64062814.x

El-Husseini, A. E., Williams, J., Reiner, P. B., Pelech, S., and Vincent, S. R. (1999). Localization of the cGMP-dependent protein kinases in relation to nitric oxide synthase in the brain. J. Chem. Neuroanat. 17, 45-55. doi: 10.1016/S08910618(99)00023-X

Erneux, C., Couchie, D., Dumont, J. E., Baraniak, J., Stec, W. J., Abbad, E. G., et al. (1981). Specificity of cyclic GMP activation of a multi-substrate cyclic nucleotide phosphodiesterase from rat liver. Eur. J. Biochem. 115, 503-510. doi: 10.1111/j.1432-1033.1981.tb0b231.x

Galati, S., D’angelo, V., Scarnati, E., Stanzione, P., Martorana, A., Procopio, T., et al. (2008). In vivo electrophysiology of dopamine-denervated striatum: focus on the nitric oxide/cGMP signaling pathway. Synapse 62, 409-420. doi: 10.1002/syn. 20510

Garris, P. A., and Wightman, R. M. (1994). Different kinetics govern dopaminergic transmission in the amygdala, prefrontal cortex, and striatum: an in vivo voltammetric study. J. Neurosci. 14, 442-450.

Garthwaite, G., Bartus, K., Malcolm, D., Goodwin, D., Kollb-Sielecka, M., Dooldeniya, C., et al. (2006). Signaling from blood vessels to CNS axons through nitric oxide. J. Neurosci. 26, 7730-7740. doi: 10.1523/JNEUROSCI.152806.2006
Garthwaite, J. (2008). Concepts of neural nitric oxide-mediated transmission. Eur. J. Neurosci. 27, 2783-2802. doi: 10.1111/j.1460-9568.2008.06285.x

Gervasi, N., Hepp, R., Tricoire, L., Zhang, J., Lambolez, B., Paupardin-Tritsch, D., et al. (2007). Dynamics of protein kinase A signaling at the membrane, in the cytosol, and in the nucleus of neurons in mouse brain slices. J. Neurosci. 27, 2744-2750. doi: 10.1523/JNEUROSCI.5352-06.2007

Goedhart, J., von Stetten, D., Noirclerc-Savoye, M., Lelimousin, M., Joosen, L., Hink, M. A., et al. (2012). Structure-guided evolution of cyan fluorescent proteins towards a quantum yield of 93\%. Nat. Commun. 3, 751. doi: $10.1038 /$ ncomms 1738

Gonon, F. (1997). Prolonged and extrasynaptic excitatory action of dopamine mediated by D1 receptors in the rat striatum in vivo. J. Neurosci. 17, 5972-5978.

Grynkiewicz, G., Poenie, M., and Tsien, R. Y. (1985). A new generation of Ca2+ indicators with greatly improved fluorescence properties. J. Biol. Chem. 260, 3440-3450.

Hepp, R., Tricoire, L., Hu, E., Gervasi, N., Paupardin-Tritsch, D., Lambolez, B., et al. (2007). Phosphodiesterase type 2 and the homeostasis of cyclic GMP in living thalamic neurons. J. Neurochem. 102, 1875-1886. doi: 10.1111/j.14714159.2007.04657.x

Hervé, D., and Girault, J.-A. (2005). "Chapter II Signal transduction of dopamine receptors," in Handbook of Chemical Neuroanatomy, Vol. 21, eds S. B. Dunnett, M. Bentivoglio, A. Björklund and T. Hökfelt, (Elsevier), 109-151. doi: 10.1016/S0924-8196(05)80006-5. Available online at: http://www.sciencedirect.com/science/article/pii/S0924819605800065

Honda, A., Adams, S. R., Sawyer, C. L., Lev-Ram, V., Tsien, R. Y., and Dostmann, W. R. G. (2001). Spatiotemporal dynamics of guanosine $3^{\prime}, 5^{\prime}$-cyclic monophosphate revealed by a genetically encoded, fluorescent indicator. Proc. Natl. Acad. Sci. U.S.A. 98, 2437-2442. doi: 10.1073/pnas.051631298

Hopper, R. A., and Garthwaite, J. (2006). Tonic and phasic nitric oxide signals in hippocampal long-term potentiation. J. Neurosci. 26, 11513-11521. doi: 10.1523/JNEUROSCI.2259-06.2006

Kawaguchi, Y. (1997). Neostriatal cell subtypes and their functional roles. Neurosci. Res. 27, 1-8. doi: 10.1016/S0168-0102(96)01134-0

Klarenbeek, J. B., Goedhart, J., Hink, M. A., Gadella, T. W., and Jalink, K. (2011). A mTurquoise-based cAMP sensor for both FLIM and ratiometric read-out has improved dynamic range. PLoS ONE 6:e19170. doi: 10.1371/journal.pone. 0019170

Le Moine, C., and Bloch, B. (1995). D1 and D2 dopamine receptor gene expression in the rat striatum: sensitive cRNA probes demonstrate prominent segregation of D1 and D2 mRNAs in distinct neuronal populations of the dorsal and ventral striatum. J. Comp. Neurol. 355, 418-426. doi: 10.1002/cne.903550308

Le Novere, N., Li, L., and Girault, J. A. (2008). DARPP-32: molecular integration of phosphorylation potential. Cell. Mol. Life Sci. 65, 2125-2127. doi: 10.1007/s00018-008-8150-y

Lin, D. T., Fretier, P., Jiang, C., and Vincent, S. R. (2010). Nitric oxide signaling via cGMP-stimulated phosphodiesterase in striatal neurons. Synapse 64, 460-466. doi: 10.1002/syn.20750

Martinez, S. E., Wu, A. Y., Glavas, N. A., Tang, X. B., Turley, S., Hol, W. G., et al. (2002). The two GAF domains in phosphodiesterase $2 \mathrm{~A}$ have distinct roles in dimerization and in cGMP binding. Proc. Natl. Acad. Sci. U.S.A. 99, 13260-13265. doi: 10.1073/pnas.192374899

Martins, T. J., Mumby, M. C., and Beavo, J. A. (1982). Purification and characterization of a cyclic GMP-stimulated cyclic nucleotide phosphodiesterase from bovine tissues. J. Biol. Chem. 257, 1973-1979.

Matsuoka, I., Giuili, G., Poyard, M., Stengel, D., Parma, J., Guellaen, G., et al. (1992). Localization of adenylyl and guanylyl cyclase in rat brain by in situ hybridization: comparison with calmodulin mRNA distribution. J. Neurosci. 12, 3350-3360.

Maurice, D. H. (2005). Cyclic nucleotide phosphodiesterase-mediated integration of cGMP and cAMP signaling in cells of the cardiovascular system. Front. Biosci. 10:1221-1228. doi: 10.2741/1614

Nikolaev, V. O., Gambaryan, S., Engelhardt, S., Walter, U., and Lohse, M. J. (2005). Real-time monitoring of the PDE2 activity of live cells: hormone-stimulated cAMP hydrolysis is faster than hormone-stimulated cAMP synthesis. J. Biol. Chem. 280, 1716-1719. doi: 10.1074/jbc.c400505200

Polli, J. W., and Kincaid, R. L. (1994). Expression of a calmodulin-dependent phosphodiesterase isoform (PDE1B1) correlates with brain regions having extensive dopaminergic innervation. J. Neurosci. 14, 1251-1261. 
Repaske, D. R., Corbin, J. G., Conti, M., and Goy, M. F. (1993). A cyclic GMPstimulated cyclic nucleotide phosphodiesterase gene is highly expressed in the limbic system of the rat brain. Neuroscience 56, 673-686. doi: 10.1016/03064522(93)90364-L

Rushlow, W., Flumerfelt, B. A., and Naus, C. C. (1995). Colocalization of somatostatin, neuropeptide $\mathrm{Y}$, and NADPH-diaphorase in the caudate-putamen of the rat. J. Comp. Neurol. 351, 499-508. doi: 10.1002/cne.903510403

Schultz, W., and Dickinson, A. (2000). Neuronal coding of prediction errors. Annu. Rev. Neurosci. 23, 473-500. doi: 10.1146/annurev.neuro.23.1.473

Stangherlin, A., Gesellchen, F., Zoccarato, A., Terrin, A., Fields, L. A, Berrera, M., et al. (2011). cGMP signals modulate cAMP levels in a compartment-specific manner to regulate catecholamine-dependent signaling in cardiac myocytes. Circ. Res. 108, 929-939. doi: 10.1161/CIRCRESAHA.110.230698

Van Staveren, W. C., Steinbusch, H. W., Markerink-Van Ittersum, M., Repaske, D. R., Goy, M. F., Kotera, J., et al. (2003). mRNA expression patterns of the cGMPhydrolyzing phosphodiesterases types 2, 5, and 9 during development of the rat brain. J. Comp. Neurol. 467, 566-580. doi: 10.1002/cne.10955

Vincent, S. R. (2000). "Chapter II Histochemistry of nitric oxide synthase in the central nervous system," in Handbook of Chemical Neuroanatomy, Vol. 17, eds H. W. M. Steinbusch, J. De Vente, and S. R. Vincent (Elsevier), 19-49. doi: 10.1016/S0924-8196(00)80056-1

Vincent, S. R., and Kimura, H. (1992). Histochemical mapping of nitric oxide synthase in the rat brain. Neuroscience 46, 755-784. doi: 10.1016/03064522(92)90184-4

Wei, J. Y., Roy, D. S., Leconte, L., and Barnstable, C. J. (1998). Molecular and pharmacological analysis of cyclic nucleotide-gated channel function in the central nervous system. Prog. Neurobiol. 56, 37-64. doi: 10.1016/S0301-0082(98) 00029-X

West, A. R., Galloway, M. P., and Grace, A. A. (2002). Regulation of striatal dopamine neurotransmission by nitric oxide: effector pathways and signaling mechanisms. Synapse 44, 227-245. doi: 10.1002/syn.10076

West, A. R., and Grace, A. A. (2000). Striatal nitric oxide signaling regulates the neuronal activity of midbrain dopamine neurons in vivo. J. Neurophysiol. 83, 1796-1808.
West, A. R., and Grace, A. A. (2004). The nitric oxide-guanylyl cyclase signaling pathway modulates membrane activity states and electrophysiological properties of striatal medium spiny neurons recorded in vivo. J. Neurosci. 24, 1924-1935. doi: 10.1523/JNEUROSCI.4470-03.2004

West, A. R., and Tseng, K. Y. (2011). Nitric oxide-soluble guanylyl cyclase-cyclic GMP signaling in the striatum: new targets for the treatment of Parkinson's disease? Front. Syst. Neurosci. 5:55. doi: 10.3389/fnsys.2011.00055

Wykes, V., Bellamy, T. C., and Garthwaite, J. (2002). Kinetics of nitric oxide-cyclic GMP signalling in CNS cells and its possible regulation by cyclic GMP. J. Neurochem. 83, 37-47. doi: 10.1046/j.1471-4159.2002. 01106.x

Xie, Z., Adamowicz, W. O., Eldred, W. D., Jakowski, A. B., Kleiman, R. J., Morton, D. G., et al. (2006). Cellular and subcellular localization of PDE10A, a striatum-enriched phosphodiesterase. Neuroscience 139, 597-607. doi: 10.1016/j.neuroscience.2005.12.042

Conflict of Interest Statement: The authors declare that the research was conducted in the absence of any commercial or financial relationships that could be construed as a potential conflict of interest.

Received: 23 July 2013; accepted: 25 October 2013; published online: 18 November 2013.

Citation: Polito M, Klarenbeek J, Jalink K, Paupardin-Tritsch D, Vincent P and Castro LRV (2013) The NO/cGMP pathway inhibits transient cAMP signals through the activation of PDE2 in striatal neurons. Front. Cell. Neurosci. 7:211. doi: 10.3389/fncel. 2013.00211

This article was submitted to the journal Frontiers in Cellular Neuroscience. Copyright (c) 2013 Polito, Klarenbeek, Jalink, Paupardin-Tritsch, Vincent and Castro. This is an open-access article distributed under the terms of the Creative Commons Attribution License (CC BY). The use, distribution or reproduction in other forums is permitted, provided the original author(s) or licensor are credited and that the original publication in this journal is cited, in accordance with accepted academic practice. No use, distribution or reproduction is permitted which does not comply with these terms. 